\title{
A CLASS OF ERGODIC TRANSFORMATIONS HAVING SIMPLE SPECTRUM
}

\author{
J. R. BAXTER
}

ABSTRACT. A class of ergodic, measure-preserving, invertible point transformations is defined, called class $S$. Any measurepreserving point transformation induces a unitary operator on the Hilbert space of $\mathfrak{L}_{2}$-functions. A theorem is proved here which implies that the operator induced by any transformation in class $S$ has simple spectrum. [It is then a known result that the transformations in class $S$ have zero entropy.]

Let $(X, \mathcal{F}, \mu)$ be a measure space, isomorphic to the unit interval with Lebesgue measure. A measurable, measure-preserving, invertible point transformation of $X$ is called an automorphism of $(X, \mathcal{F}, \mu)$. A class of automorphisms, called class $S$ for brevity, is defined below (Definition (4)). The purpose of this paper is to prove the following theorem:

(1) TheOREM. Let $\tau$ be an automorphism in class $S$. Then there exist arbitrarily small sets whose characteristic functions each generate $\mathscr{L}^{2}(d \mu)$ under the action of the unitary operator $U_{\tau}$, where $U_{\tau}$ is defined by $U_{\tau} f(\tau x)=f(x)$. In particular $U_{\tau}$ has simple spectrum.

(2) Definition. Let $H$ be a Hilbert space, $T$ a bounded normal operator on $H$. Let $v \in H$. Let $H(v)$ consist of the closure of the set of all elements of the form $P\left(T, T^{*}\right) v$, where $P\left(T, T^{*}\right)$ denotes a polynomial in $T$ and $T^{*}$. To say that a vector $v \in H$ "generates $H$ under the action of $T^{\prime \prime}$ means that $H=H(v)$.

(3) Definition. Let $\xi=\left\{A_{i} \mid 1 \leqq i \leqq m\right\}$ be a finite, ordered collection of mutually disjoint measurable sets. Then $\xi$ is called a partition. The union of the members of $\xi$ need not be the whole space. Let $\xi_{k}$ be a sequence of partitions with the property that for every measurable set $E$, there exists a sequence of sets $E_{k}$ such that each $E_{k}$ is a union of members of $\xi_{k}$, and $\mu\left(E \triangle E_{k}\right) \rightarrow 0$ as $k \rightarrow \infty$. Then it will be said that $\xi_{k} \rightarrow \epsilon$. Here $\epsilon$ denotes the partition of the whole space into one-point sets.

(4) Definition. Let $\tau$ be an automorphism of $(X, \mathcal{F}, \mu)$, $\xi=\left\{A_{i} \mid 1 \leqq i \leqq m\right\}$ a partition. If $\tau A_{i}=A_{i+1}$ for $1 \leqq i \leqq m-1, \xi$ will

Received by the editors March 30, 1970.

AMS 1969 subject classifications. Primary 2870; Secondary 4730.

Key words and phrases. Ergodic point transformation, simple spectrum, zero entropy, stacking method. 
be called a $\tau$-partition. If a sequence of $\tau$-partitions $\xi_{k}$ exists such that $\xi_{k} \rightarrow \epsilon$, then $\tau$ will be said to be in class $S$.

The automorphisms constructed in [2] and [5] are examples of class $S$ automorphisms. Some general properties of automorphisms in class $S$ are given in [1] and [3].

The first part of the proof of Theorem (1) is completed in Lemma (9), below. Some simple definitions and lemmas are given first, to simplify the proof of Lemma (9).

(5) Definition. Let $\xi=\left\{A_{i} \mid 1 \leqq i \leqq m\right\}, \eta=\left\{B_{j} \mid 1 \leqq j \leqq n\right\}$ be partitions. $\eta \leqq \xi$ will be written to mean that each member of $\eta$ is a union of members of $\xi$. If $E$ is a set, $E \leqq \xi$ will mean $\{E\} \leqq \xi$. If $m=n$ let $\rho(\xi, \eta)=\sum_{i=1}^{m} \mu\left(A_{i} \triangle B_{i}\right)$. For any $\xi$ and $\eta$, let $D(\eta, \xi)$ be the smallest number of the form $\rho\left(\eta, \eta^{\prime}\right)$, where $\eta^{\prime} \leqq \xi$. Let $D(E, \xi)=D(\{E\}, \xi)$.

Thus $\xi_{k} \rightarrow \epsilon$ means $D\left(E, \xi_{k}\right) \rightarrow 0$ as $k \rightarrow \infty$ for all measurable sets $E$.

(6) Definition. Let $\xi=\left\{A_{i} \mid 1 \leqq i \leqq m\right\}$ be a $\tau$-partition. Let $\eta$ $=\left\{B_{j} \mid 1 \leqq j \leqq n\right\}$ be a partition. Let $\tau-D(\eta, \xi)$ be the smallest number of the form $\rho\left(\eta, \eta^{\prime}\right)$, where $\eta^{\prime}=\left\{B_{\jmath}^{\prime} \mid 1 \leqq j \leqq n\right\}$ is a $\tau$-partition, $\eta^{\prime} \leqq \xi$, and also: if $I_{j}^{\prime}$ denotes the set of indices $i$ such that $A_{i} \subset B_{j}^{\prime}, 1 \leqq j \leqq n$, then $I_{j+1}^{\prime}=\left\{i+1 \mid i \in I_{j}^{\prime}\right\}, 1 \leqq j \leqq n-1$.

(7) Lemma. Let $\xi$ and $\xi^{\prime}$ be $\tau$-partitions with the same number of elements. Let $\eta$ be a partition. Then

$$
\tau-D\left(\eta, \xi^{\prime}\right) \leqq \tau-D(\eta, \xi)+\rho\left(\xi, \xi^{\prime}\right) .
$$

Proof. Follows directly from the definitions.

(8) Lemma. Let $\xi=\left\{A_{i} \mid 1 \leqq i \leqq m\right\}$ and $\eta=\left\{B_{j} \mid 1 \leqq j \leqq n\right\}$ be $\tau$ partitions. Then

$$
\tau-D(\eta, \xi) \leqq n D\left(B_{1}, \xi\right)+n \mu\left(A_{1}\right) .
$$

Proof. Let $F$ be a measurable set, $F \leqq \xi$, such that $\mu\left(B_{1} \triangle F\right)$ $=D\left(B_{1}, \xi\right)$.

Let $G$ be the set obtained by, first, removing from $F$ all sets $A_{i}$, such that $\mu\left(B_{1} \cap A_{i}\right) \leqq \frac{1}{2} \mu\left(A_{i}\right)$, and, second, removing that $A_{i}$ of largest index $i$ still remaining in $F$.

Let $I_{1}^{\prime}$ be the set of indices $i$ such that $A_{i} \subset G$. Because $\eta$ is a $\tau$ partition, it follows that any two indices in $I_{1}^{\prime}$ must differ by at least $n$, and also that the largest index in $I_{1}^{\prime}$ is less than $m-n+1$.

Let $B_{j}^{\prime}=\tau^{j-1} G, 1 \leqq j \leqq n$. Define the $\tau$-partition $\eta^{\prime}=\left\{B_{j}^{\prime} \mid 1 \leqq j \leqq n\right\}$.

It follows from the facts about $I_{1}^{\prime}$ just stated, that $\eta^{\prime} \leqq \xi$, and also that the conditions on $\eta^{\prime}$ given in Definition (6) are satisfied. Hence by definition $\tau-D(\eta, \xi) \leqq \rho\left(\eta, \eta^{\prime}\right)$. 
Furthermore, it is clear from the construction of $G$ that $\mu\left(B_{1} \Delta G\right)$ $\leqq D\left(B_{1}, \xi\right)+\mu\left(A_{i}\right)$. Since $\rho\left(\eta, \eta^{\prime}\right)=n \mu\left(B_{1} \Delta G\right)$ the lemma is proved.

(9) Lemma. Let $\xi_{k}$ be a sequence of $\tau$-partitions, $\xi_{k} \rightarrow \epsilon$. Then there exists a sequence $\eta_{k}$ of $\tau$-partitions, $\eta_{k} \rightarrow \epsilon$, such that $\eta_{k} \leqq \eta_{k+1}$, for all $k$.

Proof. By (8), it can be assumed that $\tau-D\left(\xi_{k}, \xi_{k+1}\right)<\delta_{k}, k \geqq 1$, where $\sum_{k=1}^{\infty} \delta_{k}<\infty$.

A doubly infinite sequence of $\tau$-partitions $\xi_{\boldsymbol{k}}^{r}, r \geqq 0, k \geqq 1$, will now be defined by induction.

Let $\xi_{k}^{0}=\xi_{k}, k \geqq 1$.

Having defined $\xi_{k}^{r}, k \geqq 1$, let $\xi_{\boldsymbol{k}}^{r+1}$ be some $\tau$-partition such that

$$
\tau-D\left(\xi_{k}^{r+1}, \xi_{k+1}^{r}\right)=0
$$

and

$$
\rho\left(\xi_{k}^{r}, \xi_{k}^{r+1}\right)=\tau-D\left(\xi_{k}^{r}, \xi_{k+1}^{r}\right) .
$$

Suppose that for some $r \geqq 0$, and all $k \geqq 1$,

$$
\rho\left(\xi_{k}^{r}, \xi_{k}^{r+1}\right)<\delta_{r+k} .
$$

Then since

$$
\begin{aligned}
\rho\left(\xi_{k}^{r+1}, \xi_{k}^{r+2}\right) & =\tau-D\left(\xi_{k}^{r+1}, \xi_{k+1}^{r+1}\right), \tau-D\left(\xi_{k}^{r+1}, \xi_{k+1}^{r}\right)=0, \\
\therefore \rho\left(\xi_{k}^{r+1}, \xi_{k}^{r+2}\right) & \leqq \rho\left(\xi_{k+1}^{r}, \xi_{k+1}^{r+1}\right)<\delta_{r+1+k},
\end{aligned}
$$

using Lemma (7) and the assumption.

Hence (12) holds with $r$ replaced by $r+1$. Since (12) holds for $r=0$ and all $k$, it is true for all $r, k$, by induction.

From (12),

$$
\rho\left(\xi_{k}^{r}, \xi_{k}^{r+p}\right)<\sum_{q=0}^{p-1} \delta_{q+r+k}
$$

for $r \geqq 0, p \geqq 0, k \geqq 1$.

Hence $\xi_{k}^{r}$ is a Cauchy sequence in $r$ for each $k$, and it follows that there exists a partition $\eta_{k}$ such that $\rho\left(\xi_{k}^{r}, \eta_{k}\right) \rightarrow 0$ as $r \rightarrow \infty$, for each $k \geqq 1$. It is clear that $\eta_{k}$ must be a $\tau$-partition.

Taking limits in (10) shows that $\eta_{k} \leqq \eta_{k+1}, k \geqq 1$.

Finally, since $\xi_{k} \rightarrow \epsilon$ and $\rho\left(\xi_{k}, \eta_{k}\right) \leqq \sum_{l=k}^{\infty} \delta_{l} \rightarrow 0$ as $k \rightarrow \infty$, it follows that $\eta_{k} \rightarrow \epsilon$. This completes the proof of Lemma (9).

The next lemma concerns an abstract Hilbert space $H$, and a bounded normal operator $T$. For any subspace $V$ of $H$, let $E_{V}$ denote the orthogonal projection on $V$. Suppose a sequence of elements $v_{n}$ exists, such that $v_{n} \rightarrow 0$ and $E_{H\left(v_{n}\right)} \rightarrow I$ (strongly). 
(14) Lemma. There exists a subsequence $v_{n_{k}}$ such that $v=\sum_{k} v_{n_{k}} \in H$ and $H(v)=H$.

Proof. Let $S$ be any bounded operator that commutes with $T$ and $T^{*}$. Then, for each $n, E_{H\left(v_{n}\right)} S E_{H\left(v_{n}\right)}$ commutes with $T$ and $T^{*}$ on $H\left(v_{n}\right)$. Since $T$ has simple spectrum as an operator on each $H\left(v_{n}\right)$, it can be shown using the spectral theorem, or rather, using the simplest case of the spectral representation theorem, that there exists a sequence $P_{m}^{n}$ of polynomials in $T$ and $T^{*}$ such that: $P_{m}^{n}$ $\rightarrow E_{H\left(v_{n}\right)} S E_{H\left(v_{n}\right)}$ on $H\left(v_{n}\right)$ as $m \rightarrow \infty$, and $\left\|P_{m}^{n}\right\| \leqq\|S\|$, for all $n, m$.

$H$ is clearly separable. Let $\left\{u_{1}, u_{2}, \cdots\right\}$ be a basis for $H$.

Since $E_{H\left(v_{n}\right)} \rightarrow I$, it is easy to see that sequences $n_{k}, m_{k}$ can be chosen, such that

$$
\left\|\left(P_{m_{k}}^{n_{k}}-S\right) u_{l}\right\|<1 / k, \quad 1 \leqq l \leqq k .
$$

Hence $P_{m_{k}}^{n_{k}} \rightarrow S$ as $k \rightarrow \infty$.

Thus any bounded operator $S$ which commutes with $T$ and $T^{*}$ is a strong limit of polynomials in $T$ and $T^{*}$. Since $T$ has this property it follows at once from the spectral representation theorem that $T$ has simple spectrum.

Hence it may be assumed without loss of generality that $H$ $=\&_{2}(Y, \mathcal{S}, m)$, where $m$ is a finite Borel measure on a compact subset $Y$ of the complex plane, and $T$ is defined by $T f=z f$. The elements $v_{n}$ will be written as $f_{n}$. The properties of the $f_{n}$ can now be expressed by: $\left\|f_{n}\right\|_{2} \rightarrow 0$ as $n \rightarrow \infty$, and $m\left(\left\{z \mid f_{n}(z)=0\right\}\right) \rightarrow 0$ as $n \rightarrow \infty$. To prove the lemma it is sufficient to show that a subsequence $f_{n_{k}}$ exists such that $f=\sum_{k} f_{n_{k}} \in \mathscr{L}_{2}(d m)$ and $|f|>0$ a.e. $(d m)$. This is a straightforward step, so the lemma is proved.

(15) Proof of Theorem (1). By Lemma (9), a sequence $\xi_{k}$ of $\tau$-partitions exists, such that $\xi_{k} \rightarrow \epsilon$ and $\xi_{k} \leqq \xi_{k+1}$ for all $k$. Let $\xi_{k}$ $=\left\{A_{i}(k) \mid 1 \leqq i \leqq m(k)\right\}$.

It is easy to see that a disjoint sequence of sets $E_{n}=A_{i_{n}}\left(k_{n}\right)$ can always be found.

Let $v_{n}=\chi_{E_{n}} \in \mathscr{L}_{2}(d \mu)$, let $T=U_{T}$.

Since $\xi_{k} \rightarrow \epsilon, \therefore E_{H\left(v_{n}\right)} \rightarrow I$ strongly. Clearly $v_{n} \rightarrow 0$.

By Lemma (14), there exists a subsequence $v_{n_{k}}$ such that

$$
v=\sum v_{n_{k}} \in \mathscr{L}_{2}(d \mu) \text { and } \boldsymbol{H}(v)=\mathscr{L}_{2}(d \mu) .
$$

But $v=\chi_{B}$, where $E=U_{k} E_{n_{k}}$.

Clearly $E$ can be made as small as desired, so the theorem is proved.

It is known (cf. $[6, \S 14.4]$ ), that any automorphism with simple spectrum has zero entropy. 
It may also be noted that Lemma (9) implies that the class $S$ defined here is precisely the class of those automorphisms which may be constructed by the stacking method, using single columns (cf. [4, $\S 6])$.

\section{REFERENCES}

1. M. A. Akcoglu, R. V. Chacon and T. Schwartzbauer, Commuting transformations and mixing, Proc. Amer. Math. Soc. 24 (1970).

2. R. V. Chacon, A geometric construction of measure preserving transformations, Proc. Fifth Berkeley Sympos. Math. Statist. and Probability (Berkeley, Calif., 1965/ 66), vol. II: Contributions to Probability Theory, part 2, Univ. of California Press, Berkeley, Calif., 1967, pp. 335-360. MR 35 \#3033.

3. R. V. Chacon and T. Schwartzbauer, Commuting point transformations, Z. Wahrscheinlichkeitstheorie und Verw. Gebiete 11 (1969), 277-287. MR 39 \#2939.

4. N. A. Friedman, Introduction to ergodic theory, Van Nostrand, Princeton, N. J., 1970.

5. Donald Ornstein, A mixing transformation that commutes only with its powers. (to appear).

6. V. A. Rohlin, Lectures on the entropy theory of transformations with invariant measure, Uspehi Mat. Nauk 22 (1967), no. 5 (137), 3-56=Russian Math. Surveys 22 (1967), no. 5, 1-52. MR $36 \# 349$.

University of Minnesota, Minneapolis, Minnesota 5545 